\title{
Implicancias del DR-CAFTA sobre el comercio intraindustrial de la República Dominicana
}

\author{
Por Alexis Cruz-Rodríguez y Rosalía Calvo Clúa*
}

\begin{abstract}
Resumen
Son numerosos los analistas, empresarios y demás agentes económicos que se cuestionan los efectos de los acuerdos de libre comercio sobre la economía de sus países miembros. Tras diez años de la entrada en vigor del acuerdo de libre comercio entre los Estados Unidos, la República Dominicana y los países de Centroamérica (DR-CAFTA), los efectos del mismo todavía no son del todo concluyentes y ello es debido, principalmente, a la dificultad de aislar en el escenario internacional los factores determinantes sobre la evolución del comercio y las estructuras productivas de las economías en general y, en particular, la de la República Dominicana. El análisis del comercio intraindustrial constituye una aproximación al estudio de dichos efectos. Los indicadores de comercio intraindustrial entre la República Dominicana y los miembros del DR-CAFTA utilizados muestran que durante el periodo 2007-2013 dicho comercio es principalmente relevante en el intercambio bilateral con los Estados Unidos y, especificamente, en los sectores pertenecientes al régimen de las zonas francas, agrupados como manufactura industrial.
\end{abstract}

Palabras clave: comercio intraindustrial; tratado de libre comercio.

Códigos del JEL: F14, F15.

\footnotetext{
* Departamento de Economía, Pontificia Universidad Católica Madre y Maestra (PUCMM) y Ministerio de Economía, Planificación y Desarrollo (MEPyD) de la República Dominicana. Dirección: Unidad Asesora de Análisis Económico y Social (UAAES), Ministerio de Economía, Planificación y Desarrollo, Edif. de Oficinas Gubernamentales, Bloque B, 2do Piso, Av. México Esq. Dr. Delgado, Distrito Nacional, República Dominicana. T: 809688 7000 (email: acruz@economia.gob.do.com y rcalvo@economia.gob.do). Agradecimientos: se agradecen los valiosos comentarios de los miembros de la UAAES y de los asistentes al desayuno del capítulo de economía de la Cámara Americana de Comercio.
} 


\title{
Implications of DR-CAFTA on intra-industry trade of the Dominican Republic
}

\author{
By Alexis Cruz-Rodríguez y Rosalía Calvo Clúa*
}

\begin{abstract}
There are numerous analysts, businessmen and other economic agents who question the effects of free trade agreements on the economy of its member countries. After nearly ten years of the entry into force of the free trade agreement between the United States, the Dominican Republic and Central America (DR-CAFTA), its effects are not yet fully conclusive and this is due, mainly, because the difficulty of isolating on the international stage the determinants of the evolution of trade and productive structures of the economies in general and, in particular, of the Dominican Republic. IIT analysis is an approach to the study of these effects. IIT indicators between the Dominican Republic and members of DR-CAFTA used show that during the period 2007-2013 such trade is mainly relevant in bilateral trade with the United States, and specifically in the areas belonging to the regime free zones, grouped as industrial manufacturing.
\end{abstract}

Keywords: intra-industry trade; free trade agreements.

JEL classification: F14, F15.

* Department of Economics, Pontificia Universidad Católica Madre y Maestra (PUCMM) and Ministry of Economy, Planning and Development of the Dominican Republic. Address: Advisory Unit for Economic and Social Analysis, Ministry of Economy, Planning and Development of the Dominican Republic, Edif. de Oficinas Gubernamentales, Bloque B, 2do Piso, Av. México Esq Dr. Delgado, Distrito Nacional, República Dominicana. T: 809 6887000 (email: acruz@economia.gob.do.com and rcalvo@economia.gob.do). Acknowledgements: We thank the useful comments of the members of the Advisory Unit of Economic and Social Analysis, as well as participants at the breakfast seminar of the American Chamber of Commerce. 


\section{Introducción}

A partir de la entrada en vigencia del Tratado de Libre Comercio de los países de América del Norte (NAFTA, por sus siglas en inglés) en enero de 1994, varios países de América Latina y El Caribe manifestaron su interés de negociar un acuerdo de libre comercio con los Estados Unidos, uno de sus principales socios comerciales, con miras a consolidar y profundizar los lazos comerciales. En ese sentido, en el año 2004 los países de Centroamérica y la República Dominicana firmaron un tratado con los Estados Unidos (DR-CAFTA, por sus siglas en inglés), el cual entró en vigencia en 2007.

Tras diez años de la entrada en vigencia de dicho acuerdo, los efectos del DR-CAFTA sobre el comercio, la estructura productiva y la economía en general de la República Dominicana se han analizado desde múltiples perspectivas. Los primeros trabajos sobre el impacto del DR-CAFTA en la economía dominicana provinieron de Guzmán (2005) e Isa-Contreras (2005) quienes, a un año de la firma de dicho acuerdo y sin la entrada en vigencia del mismo, analizan su impacto potencial sobre el sector industrial de la República Dominicana (Guzmán, 2005) y sobre el flujo de importaciones y las recaudaciones tributarias (Isa-Contreras, 2005).

De igual forma, Veloz (2008a), a un año de vigencia del acuerdo, analiza la competitividad de las exportaciones e importaciones dominicanas, así como los cambios en el flujo de comercio, los precios domésticos y la riqueza entre la República Dominicana y los países signatarios del mismo.

Por su parte, Villanueva (2009) evalúa el impacto de la estructura tributaria del país en los costos y la competitividad del sector exportador, a la luz del tratado de libre comercio entre los Estados Unidos, la República Dominicana y los países de Centroamérica. Asimismo, Villanueva \& Polanco (2010) valoran el impacto del acuerdo sobre los flujos de inversión extranjera directa hacia la economía dominicana. 
Similarmente, Molina et al. (2010) analizan el impacto del DR-CAFTA sobre las exportaciones dominicanas, pero desde una perspectiva microeconómica, dado que centran su análisis en el comportamiento de los exportadores. En cambio, Alemany (2011) analiza el impacto del acuerdo sobre los sectores productivos de la República Dominicana en los primeros cuatro años de su implementación. Mientras Vásquez-Ruíz, Díaz, Rivas \& Altagracia (2012) evalúan el impacto fiscal que ha tenido el DR-CAFTA sobre la economía de la República Dominicana.

A pesar de que el impacto del DR-CAFTA en la economía dominicana se ha abordado desde distintas perspectivas, ninguno de los trabajos anteriores ha considerado explícitamente el impacto del mismo sobre el comercio intraindustrial. El comercio intraindustrial se define como aquel que se presenta cuando un país importa y exporta simultáneamente bienes y servicios similares o que se encuentran clasificados dentro de una misma categoría industrial. La presencia de comercio intraindustrial es relevante ya que representa beneficios y posibilidades para los países en desarrollo y emergentes, debido a que explica en buena medida el crecimiento del comercio con base a economías de escala y diferenciación de productos.

Para el caso de una economía pequeña y abierta como es la de República Dominicana, la presencia de comercio intraindustrial puede incrementar las posibilidades de comercio internacional, la especialización y el desarrollo de largo plazo en la medida que puede suponer el incremento de ingresos, empleo y productividad. Estas últimas debido a la creación de puestos de trabajo que puede impulsar la participación en cadenas de valor, así como la mayor transferencia tecnológica.

En el presente estudio se analiza la presencia de comercio intraindustrial en los flujos de las exportaciones e importaciones de la República Dominicana con los demás miembros del DR-CAFTA durante el período 2007-2013. Para ello, se utiliza el índice de comercio intrain- 
dustrial, tanto el simple como el ponderado, desarrollado por Grubel \& Lloyd (1975). Para realizar el análisis de este índice en los países del DR-CAFTA, se consideró desagregar las partidas de los sectores seleccionados mediante la Clasificación Uniforme para el Comercio Internacional (CUCI) de la Organización de las Naciones Unidas.

El resto del trabajo está organizado de la siguiente manera: la sección 2 presenta una caracterización del comercio intraindustrial. La sección 3 muestra una breve revisión de la literatura sobre el comercio intraindustrial. La sección 4 presenta la evolución del comercio de la República Dominicana bajo el DR-CAFTA. La sección 5 presenta la metodología de medición del comercio intraindustrial. La sección 6 describe los datos utilizados y presenta un análisis de los resultados. Finalmente, la sección 7 presenta las conclusiones.

\section{Caracterización del comercio intraindustrial}

Tomando como base la teoría contemporánea del comercio exterior, el incremento en los flujos comerciales y, por consiguiente, el intercambio de doble vía que han experimentado las economías industrializadas, se ha debido en gran medida a la fragmentación vertical de la producción y, como resultado, la existencia de comercio intraindustrial. En ese sentido, la presente sección se basa en el trabajo de la Organización para la Cooperación y el Desarrollo Económico (OECD, por sus siglas en inglés). La OECD (2002) destaca las siguientes características del comercio intraindustrial:

- Los productos manufacturados especializados basados en la diferenciación y la fragmentación de la cadena de valor de los bienes presentan especialmente características de comercio intraindustrial.

- En los países en los cuales el conjunto de las importaciones y exportaciones representan más de la mitad del Producto Interno Bruto, el comercio intraindustrial es particularmente alto. 
- El comercio intraindustrial está altamente relacionado con los flujos de inversión extranjera directa y estrechamente conectado con los tratados comerciales preferenciales.

- El comercio intrafirma (entre filiales en diferentes locaciones geográficas) basado en la diferenciación o fragmentación del producto cimienta en gran medida el comercio intraindustrial.

La existencia de comercio intraindustrial es relevante desde la perspectiva del desarrollo en la medida en que, junto con otros factores determinantes del mismo, favorece la diferenciación productiva, la cual se asume como una condición muy relevante para contar con un aparato productivo que pueda sostener e incrementar tanto la demanda externa como la propia demanda interna de los países. En comparación con patrones de comercio donde la presencia de comercio intraindustrial no es tan relevante, se considera que ello no contribuye al fomento del desarrollo de esquemas productivos integrados en cadenas de valor, de manera que se limitarían las posibilidades de crecimiento del país.

\section{El comercio intraindustrial: una breve revisión de la literatura}

A la luz de las nuevas modalidades del comercio interindustrial, así como del incremento en el volumen del mismo, las teorías clásicas del comercio exterior fueron perdiendo vigencia a partir de la década de los cincuenta. En ese sentido, se destacaron los aportes de Leontief (1953) sobre el cuestionamiento de la teoría de la dotación de factores para el caso del comercio de Estados Unidos; y los aportes de Linder (1961), el cual propuso la teoría de las demandas coincidentes, según la cual los precios relativos de los bienes manufacturados son determinados en mayor medida por la demanda que por la disponibilidad de factores. De este modo se explica que los países industrializados producen y consumen los mismos bienes manufacturados, concluyendo que la teoría de 
dotación de factores no explica de manera efectiva el comercio internacional de productos.

Asimismo, Verdoorn (1960) y Balassa (1963) mostraron un incremento en el comercio de bienes similares o bienes clasificados dentro de una misma categoría industrial entre los países europeos. Además, varios estudios realizados con respecto a la creación del Mercado Común Europeo comenzaron a aportar evidencias sobre la existencia de un cada vez mayor comercio intraindustrial; por tanto, se hacía necesario encontrar respuestas teóricas a este tipo de especialización.

Una vez que se reconoció que no era posible hacer compatibles las teorías tradicionales del comercio y los escenarios de costos decrecientes en presencia de economías de escalas, al exponer los patrones de especialización industrial, una gran parte de la explicación del comercio intraindustrial entre países industrializados se centró en la existencia de la diferenciación de productos enmarcada en la teoría de la competencia monopolística (Grubel, 1967). De esta forma, los planteamientos de Smith, Ricardo, Heckscher y Ohlin, que explicaron ampliamente la racionalidad del comercio interindustrial, comenzaron a perder importancia en el análisis.

En ese sentido, los trabajos de Balassa (1966), Willmore (1972, 1974) y Grubel \& Lloyd (1975) cobraron vigencia para justificar la integración comercial y económica. Balassa (1966) utiliza datos empíricos para examinar los beneficios del establecimiento de un mercado común. Su trabajo contribuyó a un mejor entendimiento de la relevancia empírica del comercio intraindustrial como fuente concurrente del comercio internacional en bienes manufacturados. Willmore (1972, 1974), por su lado, estudió el Mercado Común Centroamericano y mostró que la mayoría de los cambios estructurales en el sector manufacturero parecen haber tomado la forma de especialización intraindustrial, es decir, la especialización en los productos diferenciados de una industria sin necesidad de abandonar la industria de altos costos. 
Por su parte, Grubel \& Lloyd (1975) plantearon métodos para medir el comercio intraindustrial y demostraron su existencia empíricamente. Para estos investigadores, los bienes manufacturados similares eran intercambiados en los países industrializados, ya que poseían mayor diferenciación en contraste con los productos primarios.

A fines de los años setenta y durante las décadas de los ochenta y noventa, se comenzaron a refinar los fundamentos teóricos que explican el comercio intraindustrial hoy día. En ese sentido, Dixit \& Stiglitz (1977) formalizaron un esquema de competencia monopolística chamberliliano que constituyó la base para el surgimiento de las teorías del comercio intraindustrial horizontal, que permitía modelar una estructura de mercado en la cual existe un gran número de empresas capaces de producir bienes diferenciados en sus características secundarias.

De igual forma, Helpman \& Krugman (1987), basados en el esquema de demanda de Dixit \& Stiglitz (1977) y en los trabajos de Krugman (1979) y Helpman (1981), desarrollaron un modelo de equilibrio integrado para predecir patrones de especialización inter e intraindustriales, considerando la competencia monopolística y la diferenciación horizontal de productos en presencia de costos decrecientes ${ }^{1}$. De un modo similar, otros investigadores hicieron importantes aportes con respecto a la separación del comercio intraindustrial horizontal y vertical (Falvey \& Kierzkowski, 1987). No obstante, a nivel empírico el debate se centró en mejorar los métodos de estimación, aunque estos no han despreciado los métodos iniciales.

Con respecto al comercio intraindustrial de la República Dominicana, solo dos estudios, hasta donde tenemos conocimiento, tratan sobre este tema. El primero de ellos, realizado por Lizardo \& Guzmán (2001), plantea una mayor incidencia del comercio intraindustrial en

\footnotetext{
${ }^{1}$ Este modelo dio inicio a la llamada "nueva visión clásica" del comercio internacional.
} 
un grupo significativo de renglones desde fines de los años noventa. Para los autores, el comercio que realiza la República Dominicana con el exterior (excluyendo el sistema de zonas francas) es esencialmente de tipo interindustrial, siendo tradicionalmente un importador de bienes intensivos en tecnología o en recursos naturales no existentes en el país. La presencia de comercio intraindustrial en el país se sustenta en el desarrollo paralelo de exportaciones e importaciones de bienes de consumo no duradero y algunas manufacturas ligeras. Los autores utilizan el índice desarrollado por Grubel \& Lloyd (1975) para mostrar que el comercio intraindustrial, en el período 1995-1999, aumentó para 57 capítulos (de los 96 capítulos que integran el Sistema Armonizado) y se redujo para 35 capítulos, mientras que se mantuvo inalterado para los 4 restantes.

El segundo estudio sobre comercio intraindustrial, realizado por la Comisión Económica para América Latina y el Caribe (2003), analiza la intensidad tecnológica de las exportaciones e importaciones de Centroamérica y la República Dominicana. El estudio cuantifica el proceso de transformación del comercio exterior de mercancías a partir de los diferentes niveles de intensidad tecnológica de sus exportaciones y de sus importaciones, lo que posibilitó determinar el valor de la balanza tecnológica de la región, el índice de intensidad tecnológica de las exportaciones, así como el índice de comercio intraindustrial ponderado para cada país. En Centroamérica y la República Dominicana el patrón comercial preponderante se inclina hacia la dependencia de un solo lado: las ramas industriales son fuertes exportadoras o alternativamente fuertes importadoras. En lo relativo al comercio intraindustrial, el estudio plantea la existencia del mismo, pero es moderado. 


\section{Evolución del comercio de la República Dominicana y el DR-CAFTA}

Si se observa la evolución del comercio entre el año 2007 (cuando entró en vigor el tratado de libre comercio) y el año 2013, se obtiene que las exportaciones de República Dominicana a los países miembros del DR-CAFTA disminuyeron en promedio -1.7\%; mientras que las exportaciones hacia el resto del mundo experimentaron un incremento de $7.3 \%$. No obstante, es importante tener en cuenta que, si se considera el año 2002, las exportaciones al resto del mundo han presentado una evolución muy dinámica y las ventas al conjunto de los países del DR-CAFTA también arrojan un crecimiento interanual negativo (-9.5\%). Destaca la relevancia de la participación del comercio con los países del DR-CAFTA sobre el comercio total, la cual durante el período 2007-2013 fue en promedio de 49.1\%.

Desde la perspectiva de los flujos comerciales de exportaciones e importaciones de República Dominicana con los países del DR-CAFTA, pueden destacarse algunas de las siguientes características durante el período 2007-2013. Primero, los saldos comerciales de la República Dominicana con los países que componen el acuerdo son deficitarios, tanto en el caso de las exportaciones nacionales netas (exportaciones menos importaciones nacionales) como de las exportaciones de zonas francas netas.

Lo anterior podría suponer en principio un efecto favorecedor para nuestros socios comerciales, con la excepción de Guatemala, donde el saldo de las exportaciones netas mejora en US\$47,268 mil; y Nicaragua, con quien República Dominicana presenta una mejoría en su saldo comercial de US\$11,144 mil. Respecto a los demás países del DR-CAFTA resulta un empeoramiento en dichos saldos (ver anexo 1, cuadro 1). 
Cuadro 1. Comercio exterior con países del DR-CAFTA en miles de USD y porCentaje del COMERCiO total de la República Dominicana.

\begin{tabular}{|c|c|c|c|c|c|c|c|c|}
\hline & 2002 & 2007 & 2008 & 2009 & 2010 & 2011 & 2012 & 2013 \\
\hline Exportaciones & $4,672,092.3$ & $4,775,580.4$ & $3,490,931.4$ & $2,978,212.0$ & $3,122,112,2$ & $3,564,345.6$ & $4,061,594.5$ & $4,230,193.8$ \\
\hline Importacio & $4,395,455.6$ & $6,414,689,4$ & $6,060,894.9$ & $5,504,330.3$ & $6,844,792.9$ & $7,507,503.6$ & $7,224,416.7$ & $7,236,206.4$ \\
\hline Total comercio a & $9,067,547.8$ & $11,190,269.7$ & $9,551,826.3$ & $8,482,542.4$ & $9,966,905.1$ & $11,071,849.2$ & $11,286,011.2$ & $11,466,400.2$ \\
\hline Exportaciones & $5,377,227,5$ & $7,247,124.2$ & $5,562,919.5$ & $4,693,263.9$ & $5,284,612.8$ & $6,376,533.5$ & $7,267,120.5$ & $7,960,986.1$ \\
\hline Importaciones de RD al mundo & $8,876,760.9$ & $13,736,042.8$ & $12,066,919,8$ & $12,015,599.9$ & $15,623,722.8$ & $17,063,515.2$ & $17,416,530.1$ & $17,575,379.1$ \\
\hline Total comercio al Mundo & $14,253,988.4$ & $20,983,166.9$ & $17,629,839.3$ & $16,708,863.8$ & $20,908,335.6$ & $23,440,048.7$ & $24,683,650,6$ & $25,536,365.2$ \\
\hline Participación del comercio con RD- & & & & & & & & \\
\hline CAFTA/Comercio total & 63.6 & 53.3 & 54.2 & 50.8 & 47.7 & 47.2 & 45.7 & 44.9 \\
\hline Saldo comercial de RD-CAFTA & $276,636.7$ & $(1,639,109.0)$ & $(2,569,963.6)$ & $(2,526,118,3)$ & $(3,722,680,8)$ & $(3,943,158.0)$ & $(3,162,822,2)$ & $(3,006,012.6)$ \\
\hline Fxportaciones de RD al RM & $705,135.2$ & $2,471,543,8$ & $2,071,988.1$ & $1,715,051.9$ & $2,162,500.6$ & $2,812,187.9$ & $3,205,526.1$ & $3,730,792,3$ \\
\hline
\end{tabular}

Fuente: Oficina Nacional de Estadísticas (ONE) y Dirección General de Aduanas (DGA).

Segundo, se ha registrado una disminución anual promedio de las exportaciones nacionales hacia los países del DR-CAFTA de $2.14 \%$ en el período analizado. Destacándose las disminuciones en las partidas de ferroaleaciones (ferroníquel) en un $11.96 \%$; aguacates, piñas y similares (5.52\%); y las demás hortalizas (1.13\%) (ver anexo 1 , cuadro 2). Con respecto a las exportaciones de zonas francas hacia los países del DR-CAFTA, se destacan las reducciones de las partidas de artículos de joyería y sus partes de metal precioso (13.15\%); trajes, conjuntos y similares de punto para hombres o niños (10.60\%); sostenes, fajas y artículos similares (8.78\%), los demás calzados $(9.27 \%)$, y el tabaco en rama (10.33\%) (ver anexo 1 , cuadro 3$)$.

Las exportaciones hacia Estados Unidos, a nivel de capítulos, experimentaron un crecimiento promedio anual de $0.5 \%$, acentuándose la disminución de las exportaciones de textiles (5.6\%); instrumentos y aparatos de óptica (8.8\%); y tabacos y sus sucedáneos (11.0\%); capítulos que, no obstante, siguen teniendo una importante participación en el total de las exportaciones dirigidas hacia Estados Unidos. A un mayor nivel de desagregación (4 dígitos), se observan disminuciones en las siguientes partidas: artículos de joyería y sus partes $(6.0 \%)$; trajes y similares de hombres (17.6\%); sostenes y similares (4.6\%); y las demás manufacturas de plásticos $(3.2 \%)^{2}$.

\footnotetext{
${ }^{2}$ Un sencillo análisis de los países competidores de República Dominicana en el mercado norteamericano muestra que, respecto al primero de los productos mencionados, los países asiáticos son los principales competidores de la partida, no obstante haber perdido la India una parte de su cuota. Los paí-
} 
Por el lado de las importaciones nacionales de la República Dominicana, provenientes de los países miembros del DR-CAFTA, estas se incrementaron en un 3.4\%. Estados Unidos continúa manteniendo su participación en el mercado de la República Dominicana durante el período analizado (91.2\% en 2013 versus $93.0 \%$ en 2007). Por capítulos, se destacan los combustibles, con un crecimiento de $117.6 \%$; y los artículos plásticos, con un crecimiento promedio de $8.4 \%$; mientras que los refrigeradores, calderas y similares disminuyeron un $0.5 \%$ (ver anexo 1 , cuadros 4 y 5).

\section{Midiendo el comercio intraindustrial}

Para evaluar el comercio intraindustrial de la República Dominicana en el marco del tratado de libre comercio entre Centroamérica, República Dominicana y los Estados Unidos, este artículo utiliza el índice, simple y ponderado, propuesto por Grubel \& Lloyd (1975). El índice simple se calcula de la forma siguiente:

$$
I G L=1-\frac{\sum / X_{i j}^{k}-M_{i j}^{k} /}{\sum\left(X_{i j}^{k}+M_{i j}^{k}\right)}
$$

donde $X_{i j}^{k}$ y $M_{i j}^{k}$ son las exportaciones e importaciones del producto de la industria o grupo $k$, del país $i$ respecto del país $j$ en un año o período dado. Alternativamente, utilizamos el índice ponderado siguiente:

$$
I G L P=\sum_{k=1}^{n} I G L^{k} * p^{k}
$$

ses asiáticos y México son los principales competidores de la partida de Trajes y similares de hombres, afectando también las exportaciones del resto de los países del DR-CAFTA donde también se observan disminuciones en sus cuotas de participación, excepto Nicaragua. Así mismo, también los países asiáticos son los principales competidores de la partida Sostenes y similares. El Salvador ha experimentado un buen crecimiento de las exportaciones de dicha partida, pudiendo haber erosionado parte del comercio de la República Dominicana, aunque el peso de las exportaciones de China e Indonesia hacia Estados Unidos es muy grande. Por último, con respecto a la partida Las demás manufacturas de plásticos, China es el principal competidor de la misma y del conjunto de los países del DR-CAFTA se destaca Costa Rica, que ha incrementado ligeramente su participación en el período analizado. 
La relevancia del índice ponderado es que se tienen en cuenta las proporciones exportadas y/o intercambiadas a nivel de cada grupo $k$ o sector considerado. De esta forma $p^{k}$ es el peso de cada producto o grupo (sector) en las exportaciones e importaciones totales del país en el sector específico. El índice de Grubel y Lloyd ponderado (IGLP) fue calculado con base en los flujos comerciales que se presentaron a nivel bilateral entre cada una de las economías de Centroamérica, los Estados Unidos y la República Dominicana. Ambos índices arrojan resultados que van desde 0 a 1 . Un resultado con un valor cercano o igual a 1 indica que el comercio se realiza entre los mismos sectores (intraindustrial), mientras que resultados cercanos a 0 indican que el comercio se realiza entre sectores de actividad diferentes (interindustrial).

Para captar la dinámica intraindustrial en las relaciones bilaterales, normalmente se utiliza una desagregación de 3 dígitos CUCI, como lo recomienda la literatura especializada (Durán \& Álvarez, 2011). Esto permite captar de manera más clara el tipo de relación comercial a nivel de industria que tiene un país con otro. Sin embargo, es importante señalar que, si se hace el mismo ejercicio a dos o a un dígito, como es el caso, el resultado será menos preciso, dado que este índice es sensible al nivel de agregación empleado para su cálculo.

Por conveniencia analítica, la literatura económica especializada recomienda que los resultados del índice se analicen siguiendo la evolución en el tiempo y el grado o nivel de su intensidad. Para ello, los resultados serán interpretados de acuerdo con los siguientes rangos propuestos por Durán \& Álvarez (2008, 2011).

$\begin{array}{ll}\text { Nivel 1: IGL }(\text { IGLP })>0.33 & \text { Indicios de comercio intraindustrial } \\ \text { Nivel 2: IGL }(\text { IGLP })>0.10<0.33 & \text { Potencial comercio intraindustrial } \\ \text { Nivel 3: IGL }(\text { IGLP })<0.10 & \text { Relaciones interindustriales }\end{array}$

A pesar de que el índice de Grubel \& Lloyd es la medida más usada para estudiar el comercio intraindustrial, el mismo presenta 
una serie de limitaciones que es importante mencionar. En primer lugar, hay que reconocer que este no tiene en cuenta la magnitud del comercio, así que debe dedicarse especial cuidado a distinguir en cuáles sectores es verdaderamente significativo un alto valor para dicho índice (Brülhart, 2002). En efecto, puede tratarse de un comercio muy incipiente en ambos sentidos, lo que arrojaría mediciones cercanas a 1 , sin que esto signifique que el comercio intraindustrial es fuerte. En segundo lugar, existe el problema de agregación. Ordenar las cuentas por sectores especificados a priori, por características predeterminadas, puede afectar el índice, por lo que se debe tener en cuenta que existe un trade-off entre el nivel de agregación (el número de dígitos de las partidas) y la facilidad para el cálculo.

\section{Datos y resultados}

Los datos compilados para el presente estudio cubren el año 2002 y el período 2007-2013. La principal razón para considerar el año 2002 es observar el comportamiento del comercio intraindustrial antes de la firma del acuerdo de libre comercio y así obtener una imagen más aproximada de las características del comercio entre los países miembros. Los datos de comercio de los países intervinientes en el acuerdo DR-CAFTA fueron obtenidos de las bases de datos de la Oficina Nacional de Estadísticas (ONE) de la República Dominicana, los cuales comprenden los valores de las exportaciones e importaciones entre estas economías, con los niveles de desagregación requeridos por el estudio. Es importante tener presente que la crisis económica de los años 2008-2009 supone un escenario complejo para analizar los efectos sobre el comercio de la República Dominicana con los países del DR-CAFTA, ya que la misma supuso la depresión de la demanda externa de nuestros socios comerciales. No obstante, el aislamiento de dicha crisis en la evolución comercial no es posible dada la metodología utilizada, por lo que quedará incorporada en el análisis del comercio intraindustrial. 
De acuerdo con los resultados, los índices de Grubel y Lloyd para el comercio intraindustrial de manufacturas no son significativos en los países miembros del DR- CAFTA, con excepción de los Estados Unidos. No obstante lo anterior, se destaca el caso de Costa Rica, donde se observa un significativo incremento de los índices IGL para la sección de los bienes manufacturados clasificados principalmente por material ${ }^{3}$. Para fines de presentación, los resultados de las ecuaciones 1 y 2 fueron multiplicados por 100 (ver anexo 2).

Dada la relevancia del comercio con los Estados Unidos, el análisis pasará a centrarse en las relaciones bilaterales entre la República Dominicana y dicha economía. En ese sentido, se analiza el grupo de manufacturas (secciones 5, 6, 7 y 8, esto es, Químicos y conexos, Manufacturas clasificadas principalmente por material, Maquinaria y equipos de transporte y Artículos manufacturados misceláneos), por considerarse que las mismas son secciones típicamente industriales, frente a otras secciones más primarias o industriales con alta presencia de recursos naturales, tales como alimentos, petróleo, combustibles, y aceites animales y vegetales. Por otra parte, se analizan las manufacturas típicamente industriales por considerarse que estas son las que tienden a generar con mayor facilidad procesos de complementación productiva $(\mathrm{CP})^{4}$.

En los gráficos 1 y 2 se observa que desde la entrada en elDR-CAFTA el comercio intraindustrial de la República Dominicana ha experimentado un comportamiento diferenciado en función del régimen considerado (nacional o de zonas francas), con respecto al comercio con los Estados Unidos. En el caso del régimen nacional, desde el año 2002 el índice correspondiente al total de los productos comerciados ha estado disminuyendo, al igual que el correspondiente a los productos manufacturados.

\footnotetext{
${ }^{3}$ No obstante lo anterior, otros países centroamericanos tienen incrementos en sus IGL en algunas secciones, pero representan montos de comercio muy pequeños.

${ }^{4}$ Algunas reflexiones de interés en torno a la complementariedad productiva pueden encontrarse en Bittencourt (2003).
} 
Gráfico 1. ÍNDice de Grubel- Lloyd PONDERAdo DEL COMERcio INTRAINDUSTRIAL ENTRE USA y RD. RÉGIMEN DE COMERCIO EXTERIOR NACIONAL, 2002 y 2007-2013

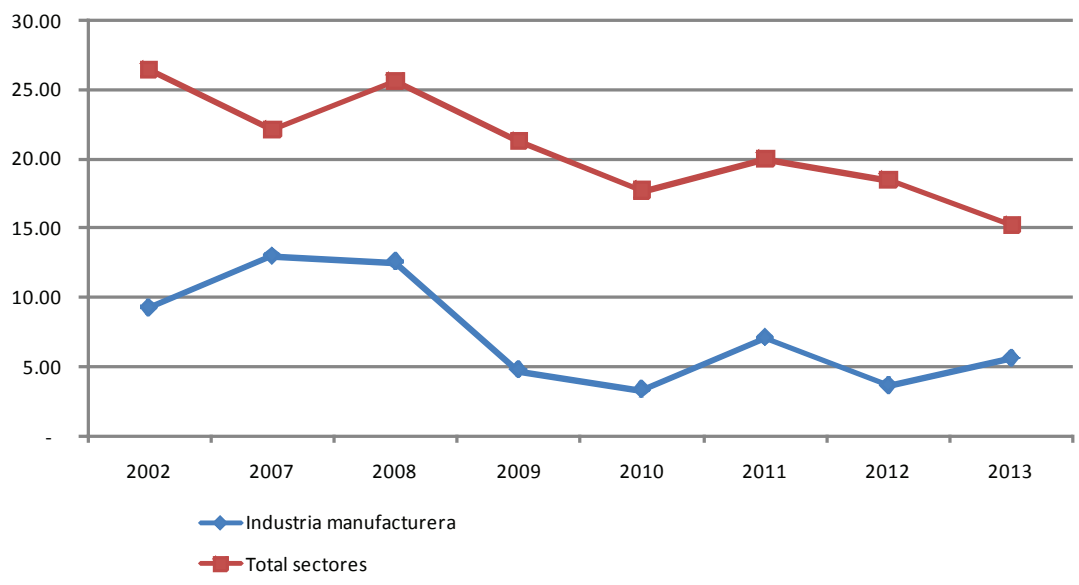

Nota. No incluye zonas francas.

Fuente. Elaboración propia con base en los datos de ONE y DGA.

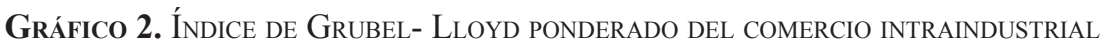
ENTRE USA Y RD. RÉGIMEN DE COMERCIO EXTERIOR DE ZONAS FRANCAS, 2002 y $2007-2013$

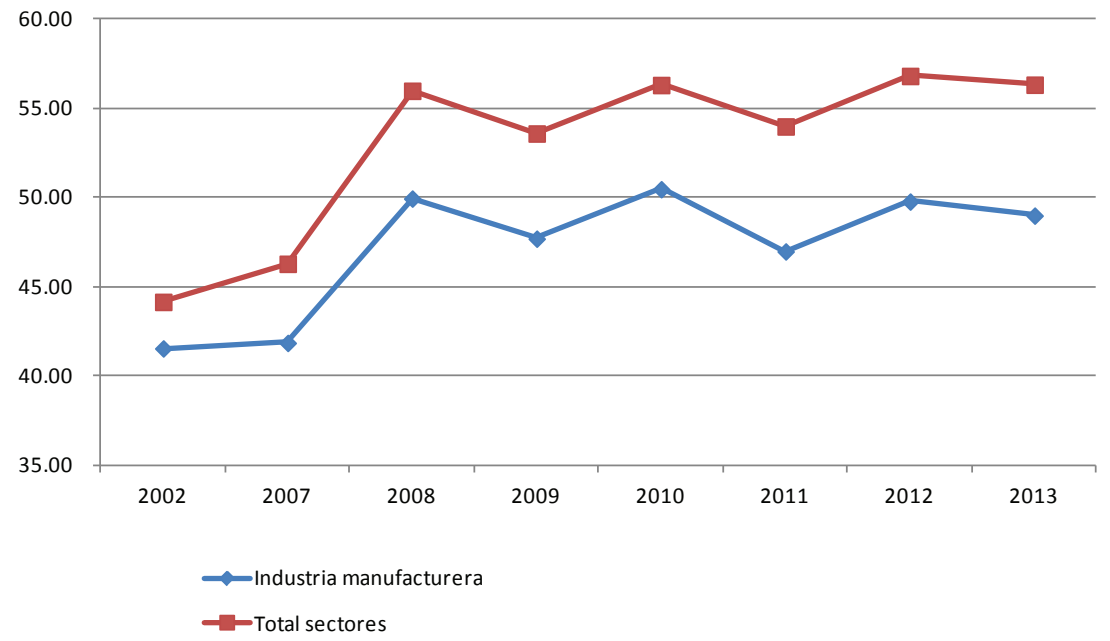

Fuente. Elaboración propia basada en datos de ONE y DGA. 
En cuanto al régimen de zonas francas, tanto los índices para el total de los productos como para el total de las manufacturas se mantiene por encima de 40 durante el período analizado -lo cual representa un valor significativo- $y$ ha mantenido una tendencia relativamente constante y ligeramente declinante, especialmente en la industria de la manufactura. En consecuencia, el análisis se concentrará en este régimen.

Con respecto a la evolución de la participación del comercio intraindustrial relevante ${ }^{5}$ de manufacturas, respecto al total de comercio, así como al total de manufacturas intraindustriales, se observa la disminución de esta desde la entrada en vigor del país en el DR-CAFTA, no habiéndose alcanzado todavía los niveles del año 2007 (cuadro 2).

Dentro del comportamiento del sector manufacturero (régimen de zonas francas) en el período analizado, se observa en el cuadro 3 una disminución en la participación de los sectores de manufacturados clasificados por material, la maquinaria y el equipo de transporte y los artículos manufacturados misceláneos. Al mismo tiempo que ocurre esta disminución en la participación en el total del comercio, se observa la disminución en los coeficientes ponderados del índice de Grubel y Lloyd. Con relación a los montos de comercio intraindustrial, es relevante el incremento observado en la sección de los químicos y productos conexos, frente a la disminución del comercio del sector de maquinaria y equipo de transporte.

Al realizar un análisis sectorial de las manufacturas, se comprueba que en 2013 el total de los sectores manufactureros con comercio intraindustrial relevante (por encima de 30) es de US\$5,560.9 millones, los cuales representan el 76.6\% del comercio bilateral de manufacturas. Si se considera únicamente el comercio equilibrado o compensado dentro de las manufacturas, se obtiene que la participación se reduce a $20.0 \%$ y que apenas ha experimentado variación desde el 2007 (ver cuadro 2).

\footnotetext{
${ }^{5}$ Con coeficiente superior a 30 (índice en base 100).
} 
Cuadro 2. Comercio Intraindustrial (CII) entre USA y RD (Manufacturas) en MILES DE USD y poRCENTAJE DEL COMERCIO TOTAL DE LA RePÚBLICA Dominicana

\begin{tabular}{|c|c|c|c|c|c|c|c|c|}
\hline & 2002 & 2007 & 2008 & 2009 & 2010 & 2011 & 2012 & 2013 \\
\hline \multicolumn{9}{|l|}{ En US\$ miles } \\
\hline $\begin{array}{l}\text { Total manufacturas intrai ndustriales } \\
\text { relevantes (con CII superior a } 30 \text { ) }\end{array}$ & $6,071,347.9$ & $6,532,066.4$ & $5,329,997.6$ & $4,292,936.5$ & $4,777,095.9$ & $5,395,323,7$ & $5,628,539.3$ & $5,560,917.8$ \\
\hline Total manufacturas intraindustriales & $7,568,481.2$ & $8,204,972.5$ & $7,226,983.5$ & $5,753,606.0$ & $6,565,842.9$ & $7,211,919.6$ & $7,380,296.1$ & $7,257,394,4$ \\
\hline Manufacturas con CII superior a 60 & $959,834.7$ & $1,681,373.5$ & $1,344,167,3$ & $977,560.8$ & $1,022,421.9$ & $1,070,716.8$ & $1,435,646.8$ & $1,451,956.6$ \\
\hline $\begin{array}{l}\text { En \% del comercio bilateral total } \\
\text { Manufacturas intrai ndustriales relevantes } \\
\text { Total comercio }\end{array}$ & 5 & & $5)^{2}$ & & & 51 & $5 \%$ & 51.5 \\
\hline Manufacturas intrai ndust triales relevantes & 00.3 & (1). & 57.1 & 0.7 .0 & & & & \\
\hline $\begin{array}{l}\text { Total manufacturas intraindustriales } \\
\text { Manufacturas con CII superior a } 60 / \text { Total }\end{array}$ & 80.2 & 79.6 & 73.8 & 74.6 & 72.8 & 74.8 & 76.3 & 76.6 \\
\hline manufacturas intraindustriales & 12.7 & 20.5 & 18.6 & 17.0 & 15.6 & 14.8 & 19.5 & 20.0 \\
\hline
\end{tabular}

Fuente. Elaboración propia basada en datos de ONE y DGA.

Entre las secciones de manufacturas con elevada concentración de comercio intraindustrial se destacan las secciones 5 y 7, Químicos y Maquinaria y equipo de transporte, respectivamente, pertenecientes al régimen de las zonas francas. Dentro de la sección 5, se destacan las partidas Preparaciones y artículos farmacéuticos, Las demás placas, láminas, hojas y tiras de plástico y Preparaciones para higiene bucal o dental, las cuales tienen importantes flujos comerciales de exportaciones de US\$195.1, US\$35.8 y US\$27.7 millones, respectivamente. En conjunto, la sección 5 presenta saldos comerciales cercanos al equilibrio. En particular, en las Preparaciones y artículos farmacéuticos y Las demás placas y tiras de plástico se destacan sus saldos comerciales favorables para la República Dominicana, por un valor de US\$172.0 y US\$15.1 millones, respectivamente.

En la sección 7 (Maquinaria y equipo de transporte) se destacan las exportaciones de zonas francas de Aparatos para corte o conexión de circuitos eléctricos (US\$364.3 millones) y Aparatos eléctricos de señalización acústica o visual (US\$34.1 millones). Las demás máquinas y aparatos de oficina (US\$21.6 millones) y los Teléfonos (US\$20.1 millones), representan el $84.7 \%$ del total exportado. Destacan los saldos comerciales favorables para la República Dominicana de estas partidas, en especial las de Aparatos para corte o conexión de circuitos eléctricos y Aparatos eléctricos de señaliza- 
ción acústica o visual por un valor de US\$155.7 y US\$28.8 millones, respectivamente.

Cuadro 3. Comercio intraindustrial por sectores CUCI entre USA y RD.

RÉGIMEN DE ZONAS FRANCAS

\begin{tabular}{|c|c|c|c|c|c|c|}
\hline \multirow[b]{2}{*}{ Secciones } & \multicolumn{2}{|c|}{$\begin{array}{l}\text { Participación del CII sobre } \\
\text { d comcrcio total (En \%) }\end{array}$} & \multicolumn{2}{|c|}{$\begin{array}{l}\text { G-LL ponderado por } \\
\text { comercio }(\operatorname{En} \%)\end{array}$} & \multicolumn{2}{|c|}{$\begin{array}{l}\text { Monto de comercio } \\
\text { intrain dustrial } \\
\text { (En miles de US\$) }\end{array}$} \\
\hline & 2007 & 2013 & 2007 & 2013 & 2007 & 2013 \\
\hline 0 . Alimentos y ani males vivos & 1.0 & 2.8 & 0.30 & 0.67 & 55,242 & 159,036 \\
\hline 1. Bebidas y Tabaco & 7.5 & 11.4 & 3.45 & 4.47 & 431,897 & 639,353 \\
\hline 2. Matcriales crudos no combustibles & 0.9 & 1.0 & 0.54 & 0.92 & 51,456 & 57,808 \\
\hline 3. Combustibles & 0.1 & 0.0 & 0.00 & 0.00 & 5,932 & 1,959 \\
\hline 4. Accites y grasas animalos y vegetales & 0.1 & 0.0 & 0.03 & 0.00 & 2,945 & 339 \\
\hline $\begin{array}{l}\text { 5. Químicos y concxos } \\
\text { 6. Manufacturados clasificados }\end{array}$ & 3.5 & 9.2 & 1.47 & 8.15 & 203,261 & 514,321 \\
\hline principalmente por material & 17.1 & 16.7 & 6.89 & 5.04 & 986,535 & 932,374 \\
\hline $\begin{array}{l}\text { 7. Maquinaria y cquipo de transporte } \\
\text { 8. Artículos manufacturados }\end{array}$ & 25.7 & 16.8 & 14.73 & 14.91 & $1,478,112$ & 937,635 \\
\hline misceláncos & 44.2 & 42.0 & 19.88 & 21.63 & $2,545,093$ & $2,352,589$ \\
\hline
\end{tabular}

Fuente. elaboración propia con base en los datos de ONE y DGA.

Desde la perspectiva sectorial de las manufacturas de zonas francas con comercio intraindustrial significativo, que se comercian entre Estados Unidos y la República Dominicana, se observa que en el año 2013 la participación más alta la tienen los Productos farmacéuticos (14.2\%), seguidos de los Instrumentos y aparatos ópticos (13.2\%). Dichos capítulos han crecido desde el año 2002 significativamente, en detrimento de las prendas textiles, las cuales al 2013 representan el $9.85 \%$ de lo comerciado, frente al $20.29 \%$ del año 2007 . Otros capítulos significativos al 2013 son los plásticos y sus manufacturas (9.7\%).

Otra característica significativa del comercio de manufacturas en el período analizado es la escasa diversificación, dado que los 11 productos seleccionados representan aproximadamente el mismo porcentaje en conjunto (alrededor de 30\%).

En cuanto a otras secciones diferentes de las manufacturas, se observa que también Bebidas y tabaco y Materiales crudos no combustibles presentan coeficientes de IGL elevados, pero cuando se ponderan por el comercio no resultan destacables. 
Gráfico 3. Comercio intraindustrial manufacturero entre USA y RD. COMPOSICIÓN SECTORIAL POR MONTOS DE COMERCIO EN PORCENTAJE (\%), AÑOS 2002, 2007 y 2013

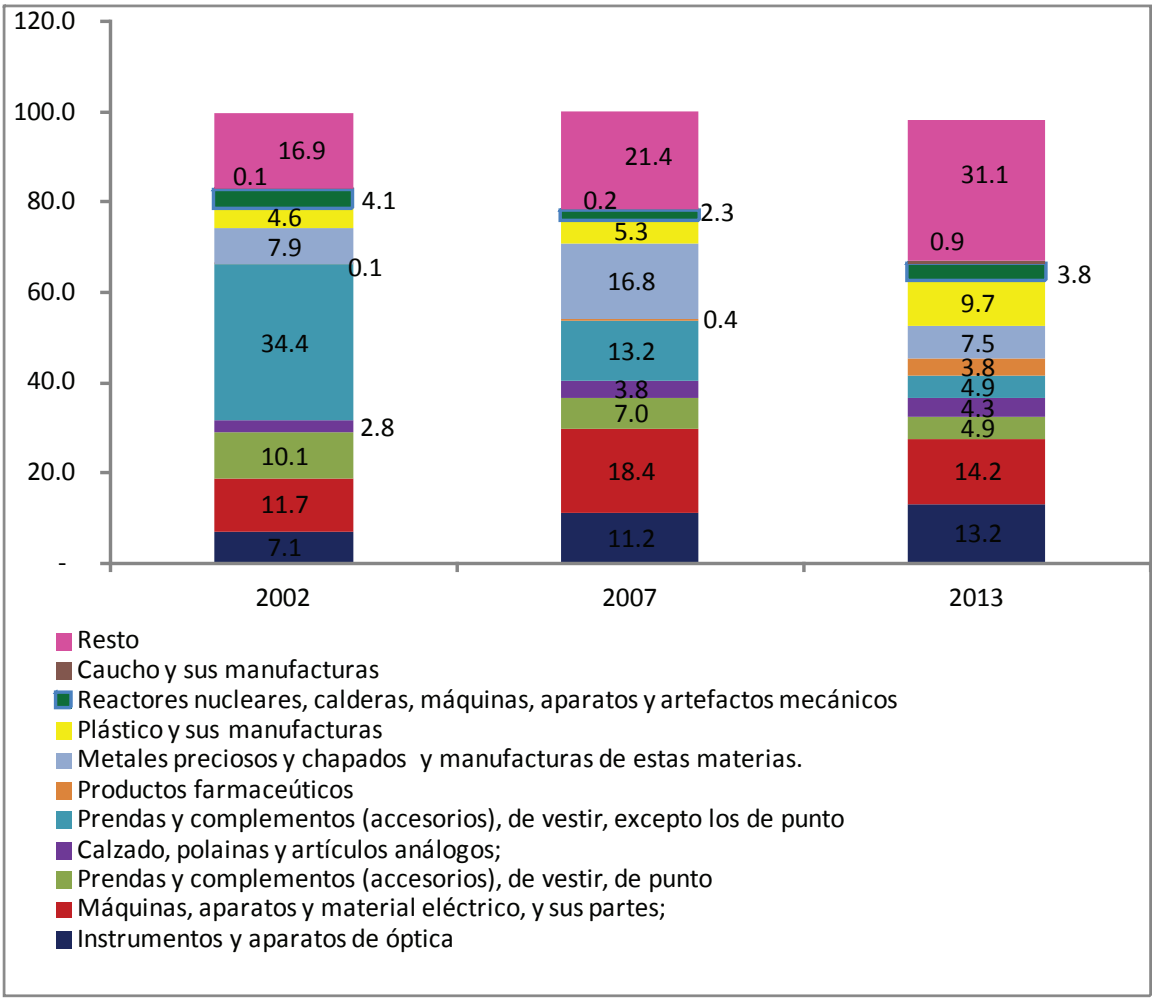

Fuente: ONE, DGA.

Tras el análisis realizado, se observa que ya que antes del acuerdo existía comercio intraindustrial de manufacturas relevante (año 2002) y la participación del comercio intraindustrial de manufacturas relevantes, tanto respecto al total de comercio como al total de manufacturas intraindustriales ha disminuido. Sí se observa el estancamiento de la participación de las manufacturas con comercio intraindustrial superior a 60 (Secciones de químicos y conexos y equipos de transporte) en el total de manufacturas con comercio intraindustrial, lo cual pudiera ser de interés en la inserción en cadenas de valor relacionadas con estas secciones -en especial químicos y conexos- que pudieran desarrollar la estructura de producción del país. 
A pesar de lo anterior, existen ciertos elementos positivos, principalmente la mejoría relativa de la sofisticación tecnológica de las exportaciones que presentan comercio intraindustrial entre Estados Unidos y República Dominicana, al migrar una parte de la producción de zonas francas de textiles hacia otras manufacturas, tales como los productos farmacéuticos, instrumentos y aparatos ópticos y los plásticos y sus manufacturas. Si se considera al conjunto de las exportaciones nacionales y de zonas francas de República Dominicana, en general, se observa un incremento de los niveles tecnológicos ${ }^{6}$.

Entre los factores que han favorecido el comercio intraindustrial en general y en particular, en el caso de República Dominicana, primero se destaca el auge del comercio intraindustrial de partes y componentes debido a los rápidos avances tecnológicos en la producción de componentes, haciendo que su fabricación se pueda realizar en diferentes lugares; segundo, las innovaciones tecnológicas en transporte y comunicaciones han facilitado la velocidad, la eficiencia y la coordinación de las transacciones productivas en diferentes puntos geográficos y, por último, las reformas de las políticas públicas, las cuales han hecho más fácil el comercio y la inversión, disminuyendo las barreras arancelarias y no arancelarias en las manufacturas. Para el caso de la República Dominicana, son especialmente importantes las políticas públicas de incentivos de zonas francas por concentrarse en el comercio intraindustrial, especialmente en este régimen de la oferta exportable ${ }^{7}$.

Los resultados obtenidos en el presente estudio se alinean, en parte, con los mostrados por Alemany (2011), donde se indica que la República Dominicana se ha beneficiado del DR-CAFTA por la garantía

\footnotetext{
${ }^{6}$ Con base en los cálculos realizados por los autores, las exportaciones con niveles tecnológicos medio y alto se incrementaron en 3.1 y 5.6 puntos porcentuales para los segmentos de exportaciones nacionales y de zonas francas, respectivamente, entre los años 2007 y 2013. No obstante, la manufactura procedente de zonas francas de alto nivel tecnológico ha perdido 11.5 puntos porcentuales en el período analizado.

${ }^{7}$ Ley Nro. 8-90 de 1990, su reglamento y sus modificaciones, Ley Nro. 139-11 de 2011 y Ley Nro. 253-12 de 2012.
} 
del acceso al mercado que ahora tiene y porque, de no haber firmado el acuerdo, hubiese estado en desventaja frente a los países que sí lo hicieron. No obstante, indica el autor, si bien es cierto que hasta el 2010 el beneficio ha sido positivo para la República Dominicana, no se debería tomar estos resultados como un indicador del desempeño futuro. Las reformas para el fomento del sector exportador son vitales para aprovechar al máximo el acuerdo comercial y poder ser competitivos en el mercado internacional.

\section{El comercio intraindustrial con Centroamérica}

No obstante haberse indicado la relevancia de Estados Unidos en el comercio intraindustrial de la República Dominicana con los países del RD-CAFTA, es importante señalar algunos resultados por país en esta subregión:

A. Guatemala

El comercio bilateral nacional entre la República Dominicana y Guatemala presenta una interesante evolución de comercio intraindustrial para la sección de Alimentos y animales, la cual es de reciente aparición (año 2013). En cuanto al comercio bilateral de zonas francas entre la RD y Guatemala, se destaca la sección de Manufacturados clasificados principalmente por material.

B. El Salvador

El comercio bilateral nacional entre la República Dominicana y El Salvador no presenta comercio intraindustrial en ninguna de sus secciones; sin embargo, el comercio intraindustrial en las zonas francas presenta cierto interés en la sección de Manufacturados clasificados principalmente por material. 


\section{Honduras}

El comercio bilateral nacional entre la República Dominicana y Honduras presenta una interesante evolución de comercio intraindustrial en las secciones de Alimentos y animales, Bebidas y tabaco y Químicos y conexos. El comercio intraindustrial en las zonas francas es relativamente relevante en las secciones de Bebidas y tabaco, Químicos y conexos y Maquinaria y equipos de transporte.

D. Costa Rica

El comercio bilateral nacional entre la República Dominicana y Costa Rica en lo que se refiere al comercio intraindustrial es de interés en la sección de Manufacturados clasificados principalmente por material. En lo que respecta al comercio intraindustrial en las zonas francas, este es de cierta importancia en esta sección también, a pesar de haber presentado una importante disminución en el año 2013.

E. Nicaragua

El comercio intraindustrial presente en el comercio bilateral nacional entre la República Dominicana y Nicaragua es de importancia en la sección de Químicos y conexos. En lo concerniente al comercio intraindustrial en las zonas francas, este no presenta ninguna sección de interés.

\section{Consideraciones finales}

El comercio intraindustrial representa beneficios y posibilidades para los países en desarrollo y emergentes. Los resultados obtenidos en el presente estudio muestran que, durante el período 2007-2013, el comercio intraindustrial es relevante en el intercambio bilateral con los Estados Unidos, particularmente en los sectores pertenecientes al segmento de las zonas francas, agrupados como manufactura industrial. Sin embargo, no se observa igual relevancia con el resto de los países 
miembros del DR-CAFTA, lo cual pudiera ser explicado por la menor integración existente entre dichos países y la República Dominicana, fruto de los niveles de competencia con respecto a Estados Unidos.

A pesar de ello, se puede decir que el DR-CAFTA ha ayudado a potenciar el comercio intraindustrial y, por lo tanto, ha contribuido y puede seguir contribuyendo al desarrollo productivo de la República Dominicana. Como se ha podido constatar, la diversificación en los niveles tecnológicos de las exportaciones también ha impulsado el desarrollo de nuestro aparato productivo, fomentando el incremento del potencial comercial, la especialización internacional, así como el crecimiento del ingreso, la productividad y el empleo, lo cual es posible constatar en el desarrollo de las zonas francas.

No obstante, queda mucho camino por recorrer. Es importante considerar, que los resultados obtenidos son tanto fruto de las sinergias generadas mediante la firma del acuerdo, como de los efectos incentivadores otorgados por las facilidades de la Ley de zonas francas.

Por último, es importante destacar la relevancia de una política industrial y comercial en general para alcanzar mayores niveles de desarrollo productivo. No todo termina con la firma de acuerdos comerciales como el DR-CAFTA analizado.

Para efectos de un estudio posterior a un nivel de mayor profundidad, ya sea de una o varias de las secciones seleccionadas, puede ampliarse el análisis de los productos considerados dentro de cada sección estudiada a nivel de 4 dígitos, según el Sistema Armonizado de Designación y Codificación de Mercancías.

\section{Referencias}

Alemany, N. J. (2011). Evaluación del impacto del DR-CAFTA en los sectores productivos de la República Dominicana. Santo Domingo, República Dominicana: Mimeo, USAID. 
Balassa, B. (1963). An empirical demonstration of classical comparative cost theory. The Review of Economics and Statistics, 45(3), 231-238.

Balassa, B. (1966). Tariff reductions and trade in manufactures among the industrial countries. The American Economic Review, 56(3), 466-473.

Bittencourt, G. (2003). Complementación productiva industrial y desarrollo en el Mercosur. Oportunidades y límites para la acción municipal. Documento de trabajo preparado para MERCOCIUDADES. Departamento de Economía de la Facultad de Ciencias Sociales de la República de Uruguay.

Brülhart, M. (2002). Marginal intra-industry trade: Towards a measure of non-disruptive trade expansion. En Lloyd, P. J. y HyunHoon (eds): Frontiers of Research on Intra-Industry Trade. Palgrave-Macmillan: London.

Comisión Económica para América Latina y El Caribe. (2003). Intensidad tecnológica del comercio de Centroamérica y la República Dominicana. Unidad de Comercio Internacional, Sede Subregional de la CEPAL en México, 24 de diciembre.

Dixit, A. \& Stiglitz, J. (1977). Monopolistic competition and optimum product diversity. The American Economic Review, 67(3), 297-308.

Durán, J. E. \& Álvarez, M. (2008). Indicadores de comercio exterior y política comercial: mediciones de posición y dinamismo comercial. Documento de Proyecto. Comisión Económica para América Latina y el Caribe.

Durán, J. E. \& Álvarez, M. (2011). Manual de comercio exterior $y$ política comercial: Nociones básicas, clasificaciones $e$ 
indicadores de posición y dinamismo. Documento de Proyecto. Comisión Económica para América Latina y el Caribe.

Falvey, R. \& Kierzkowski, H. (1987). Product quality, intra-idustry trade and (im)perfect competition. En Kierzkowski, H. (ed.): Monopolistic competition and international trade. Oxford: Basil Backwell.

Grubel, H. G. (1967). Intra-industry specialization and the pattern of trade. The Canadian Journal of Economics and Political Science, 33(3), 374-388.

Grubel, H. G. \& Lloyd, P. J. (1975). Intra-industry trade the theory and measurement of international trade in differentiated products. New York: Wiley.

Guzmán, R. (2005). Evaluación del impacto potencial del CAFTA-DR sobre el sector industrial dominicano. Santo Domingo, República Dominicana: Mimeo, USAID.

Helpman, E. (1981). International trade in the presence of product of differentiation economies of scale and monopolistic competition: A Chamberlin-Heckscher-Ohlin approach. Journal of International Economics, 11(3), 305-340.

Helpman, E. \& Krugman, P. (1987). Market structure and foreign trade: Increasing return, imperfect competition and the international economy. (Edición revisada). Cambridge: MIT Press.

Isa-Contreras, P. (2005). El impacto fiscal del acuerdo de libre comercio con los Estados Unidos. Texto de Discusión No.3. Secretariado Técnico de la Presidencia.

Krugman, P. (1979). Increasing returns, monopolistic competition and international trade. Journal of International Economics, 9(4), 469-480. 
Leontief, W. (1953). Domestic Production and Foreign Trade; The American capital position re-examined. Proceedings of the American Philosophical Society, 97(4), 332-349.

Lancaster, K. (1989). Intra-industry trade under perfect monopolistic competition. Journal of International Economies, 10, 151-175.

Linder, B. S. (1961). An Essay on Trade and Transformation. Primera edición. Uppsala: Almquist \& Wiksell.

Lizardo, M. \& Guzmán, R. M. (2001). Patrones de integración a la economía global: ¿Qué comercializa América Latina? ¿Qué hacen sus trabajadores? El Caso de la República Dominicana. República Dominicana: Mimeo.

Molina, A. C.; Bussolo, M. \& Iacovone, L. (2010). The DR-CAFTA and the extensive margin: A firm-level analysis. Policy Research Working Paper No.5340. The World Bank.

Organization for Economic Cooperation and Development (OECD). (2002). A recent discussion of the growing importance and characteristics of intraindustry trade. En OECD (eds): Intraindustry and intrafirm Trade and the Internationalisation of Production. Economic Outlook, No. 71.

Vásquez-Ruíz, H.; Díaz, R.; Rivas, R. \& Altagracia, A. (2012). Impacto Fiscal del DR-CAFTA en la República Dominicana. Documento de trabajo, Banco Central de la República Dominicana.

Veloz, A. (2008a). Evaluación del desempeño de la República Dominicana en el acuerdo de libre comercio con Estados Unidos y Centro América (DR-CAFTA). Santo Domingo, República Dominicana: Mimeo, USAID. 
Veloz, A. (2008b). Metodología para evaluar el desempeño de la República Dominicana en el DR-CAFTA. Mimeo, USAID. Santo Domingo, República Dominicana.

Verdoorn, P. J. (1960). The intra-block trade of Benelux. En Robison, E. (ed.): Economic consequence of the size of nations. Londres: Macmillan.

Villanueva, B. (2009). Competitividad e impuestos: un análisis del impacto del sistema tributario sobre los costos del sector exportador a la luz del DR-CAFTA. Santo Domingo, República Dominicana: Mimeo, USAID.

Villanueva, B. \& Polanco, L. (2010). El DR-CAFTA y la inversión extranjera directa en la República Dominicana. Santo Domingo, República Dominicana: Mimeo, USAID.

Willmore, L. N. (1972). Free trade in manufactured among developing countries: The Central American experience. Economic Development and Cultural Change, 20(4), 659-670.

Willmore, L. N. (1974). The pattern of trade and specialization in the Central American Common Market. Journal of Economics Studies, 1(2), 113-134. 